\title{
Assessment of Outcomes of Spine Surgery in Indian Athletes Involved in High-End Contact Sports
}

\author{
Kunal Shah ${ }^{1}$, Anuj Bharuka ${ }^{2}$, Akshay Gadiya ${ }^{2}$, Abhay Nene ${ }^{2}$ \\ ${ }^{1}$ Department of Spine Surgery, Pain and Spine Clinic, Mumbai, India \\ ${ }^{2}$ Department of Spine Surgery, Lilavati Hospital and Research Center, Mumbai, India
}

Study Design: This study was a retrospective analysis.

Purpose: This study aimed to analyze the functional outcome following spinal surgery in elite athletes using return-to-play criteria.

Overview of Literature: Spinal ailments are relatively common in athletes and are bound to increase due to the ever-growing popularity of contact sports. An elite athlete is highly motivated to make a rapid recovery and return to full participation in sports. Although the criteria for diagnosis and surgical treatment of various spinal disorders in athletes have been well defined in the literature, there is no clear definition about the factors determining the return to play in athletes.

Methods: This study was a retrospective analysis of the data of 10 elite athletes who underwent spinal surgery for symptomatic degenerative disorder of the spine. Eight patients underwent lumbar spine surgery (two patients of microdiscectomy and six patients of fusion), and the remaining two patients underwent cervical spine surgery (one each anterior cervical discectomy and fusion and anterior cervical disc replacement). Outcome measures were investigated using return-to-training and return-to-sports criteria, as indicated by the length of time between surgery and return to competitive sports as parameters.

Results: Of the 10 patients, eight were males and two were females. The average age of the patients at the time of surgery was 32.4 years (range, 25-41 years). All patients returned to active participation of their sports. The average time for return to training was 7.3 weeks (range, 3-12 weeks). The average time for return to sports was 45.6 weeks (range, 36-72 weeks), and the average follow-up period was 59 months (range, 27-120 months).

Conclusions: Spine surgery in an elite athlete involved in contact sports is safe and effective. Currently, there is a lack of standardized guidelines for return to sports after spine injuries. An athlete needs to be symptom-free, with full range of motion and full strength before returning to sports.

Keywords: Athletes; Spine surgery; Functional outcome; Return to sport

\section{Introduction}

Spinal injuries are relatively common among athletes and are bound to increase due to the ever-growing popularity of contact sports. Low back pain (LBP) is one of the most common complaints encountered in $>80 \%$ of the general population at some point in life [1]. Athletes also commonly face this problem, with the incidence rates being almost $30 \%$ over the course of their career [2]. LBP accounts for one of the most common reasons for

Received Dec 7, 2019; Revised Feb 9, 2020; Accepted Feb 25, 2020

Corresponding author: Kunal Shah

Department of Spine Surgery, Pain and Spine Clinic, 101 Excel Arcade, LBS Road, Ghatkopar West, Mumbai-400086, India

Tel: +91-9930731911, E-mail: orthokunal@yahoo.com 
absenteeism from sports. Spine injuries occurring in an elite athlete may be either catastrophic (burst fractures causing quadriparesis/quadriplegia) or noncatastrophic (minor sprains, radiculopathy, transient myelopathy), which require further investigation before intervention. The majority of patients show improvement with conservative management, but those who do not get better may require surgery. The criteria for the diagnosis and surgical treatment of various spinal disorders in athletes have been well defined, and the majority of existing literature has described the outcomes of spinal surgery in American football players [3-5]. All these studies highlight the beneficial effects of surgical treatment in this special cohort. Similarly, athletes involved in cricket, wrestling, or marathon running have high physical demands for sporting activities. An elite athlete is highly motivated to make a rapid recovery and return to full participation in sports. However, the factors determining the return to play in athletes have not been clearly defined. Successful return to play for professional athletes at their preinjury level of performance is important for their livelihood. Outcome measures such as visual analog scales, the Oswestry Disability Index, and the Short Form-36 that are used for the general population are not applicable to athletes who are interested in returning to their sports and on career longevity. Criteria involving return-to-play rates, career length, and performance-based outcomes after surgical treatment are important for professional athletes.

\section{Materials and Methods}

In this retrospective analysis, 10 consecutive patients involved in high-end impact sports diagnosed with degenerative spine pathology requiring surgical intervention between April 2009 and April 2017 were investigated. Eight patients underwent lumbar spine surgery (two patients of microdiscectomy and six patients of fusion), and two patients underwent cervical spine surgery (one patient underwent anterior cervical discectomy and fusion $[\mathrm{ACDF}]$ and the other underwent anterior cervical disc replacement). All patients had a diagnosis of degenerative spinal pathology on plain radiographs and magnetic resonance imaging. Instability was identified based on evidence of dynamic sagittal translation of $\geq 5 \mathrm{~mm}$ and/ or an angulation of $\geq 10^{\circ}$ on flexion-extension views in both cervical and lumbar spine. All patients underwent the nonoperative treatment for appropriate duration and were offered a surgical treatment in view of persistent disability. All patients had stopped playing their sports and were unable to return to play due to severe pain. Patients with lumbar and cervical spine pathology experienced significant axial spinal pain and radicular symptoms. Surgery was indicated when patients fulfilled the following criteria: pathology affecting their peak performance, recurrent episodes causing inability to participate in their sport, and failure of conservative management for at least 6 weeks. Outcome measures were evaluated using returnto-training and return-to-sports criteria, as indicated by the length of time between surgery and return to competitive sports as parameters.

Lumbar microdiscectomy was performed in two patients via a standard unilateral approach. Six patients underwent lumbar interbody fusion by transforaminal approach. Of the six patients, one was operated twice before the final surgery (once for microdiscectomy and once for laminectomy) and experienced significant back pain and leg pain. Cervical spine fusion and cervical disc replacement were performed using the anterior cervical approach. Anterior reconstruction was done using interbody cage and reconstruction plate. No patient had any postoperative neurological weakness. Table 1 shows the epidemiological details of the patients and the details of the surgery.

All patients were carefully monitored postoperatively along with their respective coach and training staff. Each patient received a strict and supervised intensive rehabilitation program as follows. Table 2 describes the detailed stages and the protocol for rehabilitation, which we followed for our athletic patients. This protocol was followed for both fusion and disc replacement patients; however, the duration for each stage was individualized depending on the pain tolerance. We delayed the same rehabilitation protocol by almost 6 weeks for microdiscectomy patients. Criteria for return to sports were recovery of range of motion, full strength and endurance to prevent further injury, ability to demonstrate preinjury level of performance, and complete resolution of preoperative pain. One patient (case 10) had aseptic discitis that caused a delay in rehabilitation that was managed nonsurgically with activity restriction and anti-inflammatory medications. None of the patients had major complications requiring revision surgery. The Institutional Review Board approval was not sought as this was retrospective analysis of our operated patients. All patients were informed and consented for 
inclusion in the study.

\section{Results}

Of the 10 patients, eight were males and two were females. The average age of the patients at the time of surgery was 32.4 years (range, $25-41$ years). Patients had experienced an average of 4.5 months of pain (range, 1.5-24 months) before their initial presentation. None of them had any neurological deficit. All patients returned to active participation of their sports, and the average time for return to training was 7.3 weeks (range, 3-12 weeks). The average time for return to training for microdiscectomy patients was 11 weeks and that for patients who underwent fusion and replacement was 6 weeks.

The average time for return to sports was 45.6 weeks (range, 36-72 weeks). The average time for return to con- tact sports for patients undergoing microdiscectomy was 22 weeks and that for patients undergoing fusion and replacement was 12 weeks. Table 1 lists the average time for return to sports for individual sports. The average followup duration was 59 months (range, 27-120 months).

\section{Discussion}

Sports professionals are special people because they have very high levels of primary fitness; they have strong willingness to return to function, require aggressive and structured rehabilitation, and have relatively short-term demands. Spinal ailments, especially lower back pain, are one of the most common reasons of missed playing time [2]. Due to the ever-increasing number of general population involved in high-demand contact sports, there has been an increase in the awareness about spinal disorders

Table 1. Details of patients

\begin{tabular}{|c|c|c|c|c|c|c|c|}
\hline No. & Sports & Diagnosis & Surgery & $\begin{array}{l}\text { Return to } \\
\text { training (wk) }\end{array}$ & $\begin{array}{l}\text { Return to contact } \\
\text { sports (mo) }\end{array}$ & $\begin{array}{l}\text { Follow-up } \\
\text { (mo) }\end{array}$ & $\begin{array}{l}\text { Reintervention/ } \\
\text { complications }\end{array}$ \\
\hline 1 & Marathoner & L4-5 PID & Microdiscectomy & 12 & 5 & 75 & None \\
\hline 2 & Cricketer & L4-5 PID with instability & L4-5 fusion & 6 & 3 & 60 & None \\
\hline 3 & Marathoner & L3-5 PID with instability & L3-5 fusion & 6 & 3 & 52 & None \\
\hline 4 & Cricket & Disc disease & L4-5 fusion & 6 & 3 & 27 & None \\
\hline 5 & Tennis & Instability & Redo L3-5 fusion & 6 & 3 & 120 & None \\
\hline 6 & Marathoner & L4-5 PID & Microdiscectomy & 10 & 6 & 105 & None \\
\hline 7 & Wrestling & C5-6 PID & $\begin{array}{l}\text { Anterior cervical discectomy and } \\
\text { fusion }\end{array}$ & 6 & 3 & 36 & None \\
\hline 8 & Marathoner & Disc disease & Anterior cervical disc replacement & 6 & 3 & 45 & None \\
\hline 9 & Marathoner & Instability & L3-5 fusion & 6 & 3 & 41 & None \\
\hline 10 & Marathoner & L3-4 listhesis & L3-4 fusion & $4 \mathrm{mo}$ & 6 & 29 & $\begin{array}{l}\text { Aseptic discitis immediate } \\
\text { postoperative }\end{array}$ \\
\hline
\end{tabular}

PID, prolapsed intervertebral disc.

Table 2. Rehabilitation protocol

Rehabilitation protocol

\section{Contents}

\begin{tabular}{ll} 
Early protected mobilization (1-3 wk) & $\begin{array}{l}\text { 1. Pain management and physical modalities (hot and cold packs) } \\
\text { 2. Gradual mobilization and range of motion: (1) maintaining neutral spine; (2) low impact aerobics; (3) protected walking and } \\
\text { staircase climbing }\end{array}$ \\
\begin{tabular}{ll} 
Dynamic stabilization (4-6 wk) & $\begin{array}{l}\text { 1. Isometric strengthening (back extensor, abdominal, gluteal) } \\
\text { 2. Low impact endurance training }\end{array}$ \\
\hline Return to training (6-12 wk) & $\begin{array}{l}\text { 1. Isotonic strengthening } \\
\text { 2. Active full range of motion } \\
\text { 3. Sports specific training }\end{array}$ \\
\hline Return to sports (after $3 \mathrm{mo})$ & Active participation in high impact sports \\
\hline Maintenance & Regular exercises, maintaining fitness, continuation of trunk stabilization program and follow-ups
\end{tabular} \\
\hline
\end{tabular}


and their prompt management in this special cohort of population. Furthermore, spinal injuries have psychosocial and financial implications in an elite athlete [6]. The livelihood of professional athletes is solely dependent on the ability to perform in their respective sports. Spinal injuries may result in poor performance, shortened career, or absolute inability to perform. Therefore, spinal ailments in an athlete should not only be investigated thoroughly but also be managed promptly with an aim to early return to sport. The longevity of the career of an elite athlete is dependent not only on the good surgical technique but also on the good functional recovery following spinal surgery.

More recently, clinical studies have focused on return to play and sport-specific, performance-based outcome measures after treatment for lumbar spine injury. Nonetheless, there is substantial variability in the published "return-toplay" criteria, which are almost exclusively derived from authors' expert opinion and experience. For professional athletes, successful return to sports after lumbar discectomy has been well documented and has become the expected norm.

Similar to lumbar sprains, lumbar disc herniation in an elite athlete is commonly managed using nonoperative modalities and physical rehabilitation. Therefore, a multidisciplinary team approach is required for achieving a successful outcome. It has been reported that $>90 \%$ of players respond well to nonoperative treatment, and the majority of patients return to sports within 6 weeks of onset [3]. Patients who do not respond to nonoperative treatment and have worsening neurological deficits with involvement of sphincters require surgery.

A patient with lumbar disc herniation can be treated surgically either by lumbar microdiscectomy or by lumbar fusion, modalities that have good functional outcome in the general population. Various studies performed among the general population have suggested the favorable outcome of surgery over nonoperative treatment $[7,8]$; however, these results cannot be generalized to the cohort of elite athletes. Parameters such as return to sports, career longevity, and performance aptly measure the outcomes of various interventions in this cohort. The Professional Athlete Spine Initiative was a retrospective cohort study of 342 professional athletes conducted from 1972 to 2008 who were diagnosed with lumbar disc herniations [4]. Of 342 patients, 226 underwent lumbar discectomy, whereas 116 underwent conservative treatment. Approximately
$81 \%$ (184 of 226 ) of the operated patients returned to play at an average time of 3.3 years following surgery. Approximately $62.3 \%$ of the players were expected to remain active for approximately 2 years following treatment. The study had concluded that National Football League (NFL) players undergoing lumbar microdiscectomy had significantly longer career lengths than players who were treated nonoperatively when compared with other sports cohorts. Another study conducted by Hsu [5] demonstrated the potential beneficial effect of surgery for lumbar disc herniation compared with conservative management. Returnto-play rates between operative and nonoperative groups were $78 \%$ versus 59\%, respectively. The author concluded that players treated operatively played significantly more games postoperatively than those treated nonoperatively. Similar results were reported by Anakwenze et al. [9] in their study. In contrast, a descriptive study reported by Schroeder et al. [10], evaluated 87 ice hockey players to determine performance-based outcomes following nonoperative and surgical treatment for lumbar disc herniations. Analysis of posttreatment results demonstrated that there was no significant difference in performance scores between the nonoperative and surgical groups. Children with disc pathologies behave differently compared with adults. They do not respond well to conservative treatment and are more concerned about return-to-play following lumbar discectomy [11]. A study conducted by Wang et al. [12] reported similar results as those of the present study for lumbar discectomy in athletes. Of the 10 college athletes who underwent single-level discectomy, nine returned to preinjury level of competition. However, the authors did not describe the time to return to sports or the level of disability at return to sports. In the present study, two of 10 patients underwent lumbar microdiscectomy; both patients were marathon runners. Both of them returned to training within 12 and 10 weeks and returned to competitive marathon running within 5 and 6 months, respectively. Both athletes returned to their preinjury level of sports. Fig. 1 illustrates the case of a marathon runner who had L5/S1 prolapsed intervertebral disc (Fig. 1A). He underwent L5/S1 microdiscectomy. Follow up scan show adequate deompression (Fig. 1B).

Till date, there has been a paucity of literature on lumbar fusion for degenerative lumbar spine pathologies among professional athletes. Shifflett et al. [13] reviewed 34 golfers undergoing a fusion procedure for degenerative pathologies of the lumbar spine. Approximately 65\% 


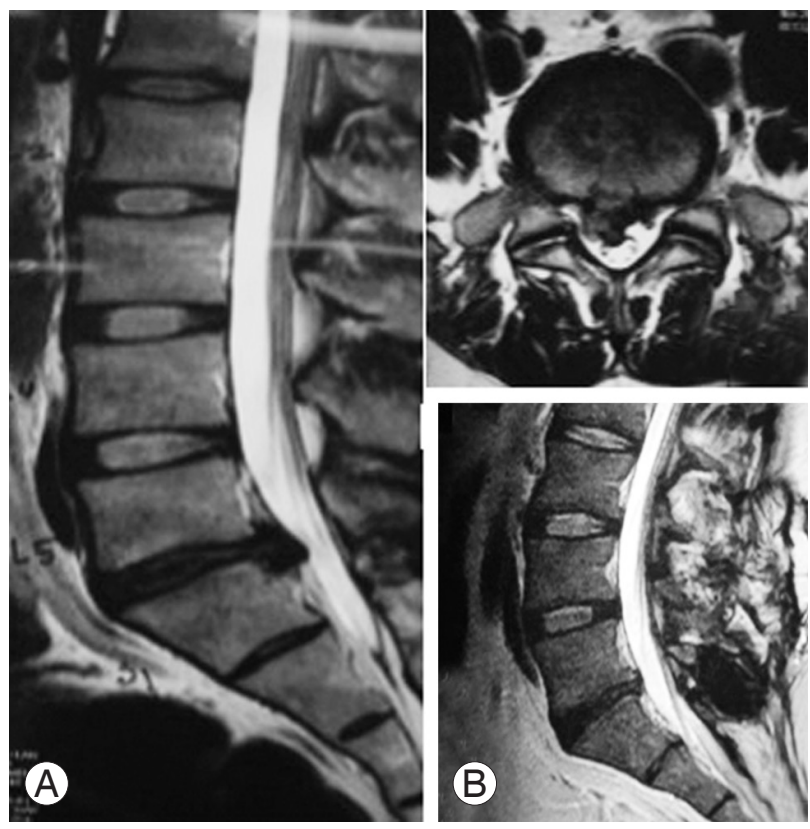

Fig. 1. (A) Preoperative T2-weighted MRI showing L5-S1 disc herniation of a patient. (B) Postoperative T2-weighted MRI sagittal section of the same patient. MRI, magnetic resonance imaging. of the patients returned to practice, and $52 \%$ of them returned to course play within 1 year of surgery. Approximately $77 \%$ of the patients were able to play the same amount of golf or more than that before the fusion surgery. In another series, Schroeder et al. [10] reported about 8 hockey players who underwent single-level lumbar spinal fusion. There was no significant difference in the number of games played per season or the performance score either before or after the treatment. All the eight players who underwent a lumbar fusion were able to successfully return to play. Furthermore, the authors suggested that a lumbar fusion is compatible with return to play in the National Hockey League, which is in contrast to other professional sports. Bouras and Korovessis [14] in their systemic review suggested that return to play following fusion surgery varies from 6 to 12 months with prohibition in collision sports. Return to play was largely dependent on specific sport activity. In the present study, six of 10 patients underwent lumbar fusion. Of these six patients, three were marathon runners, two were cricket-
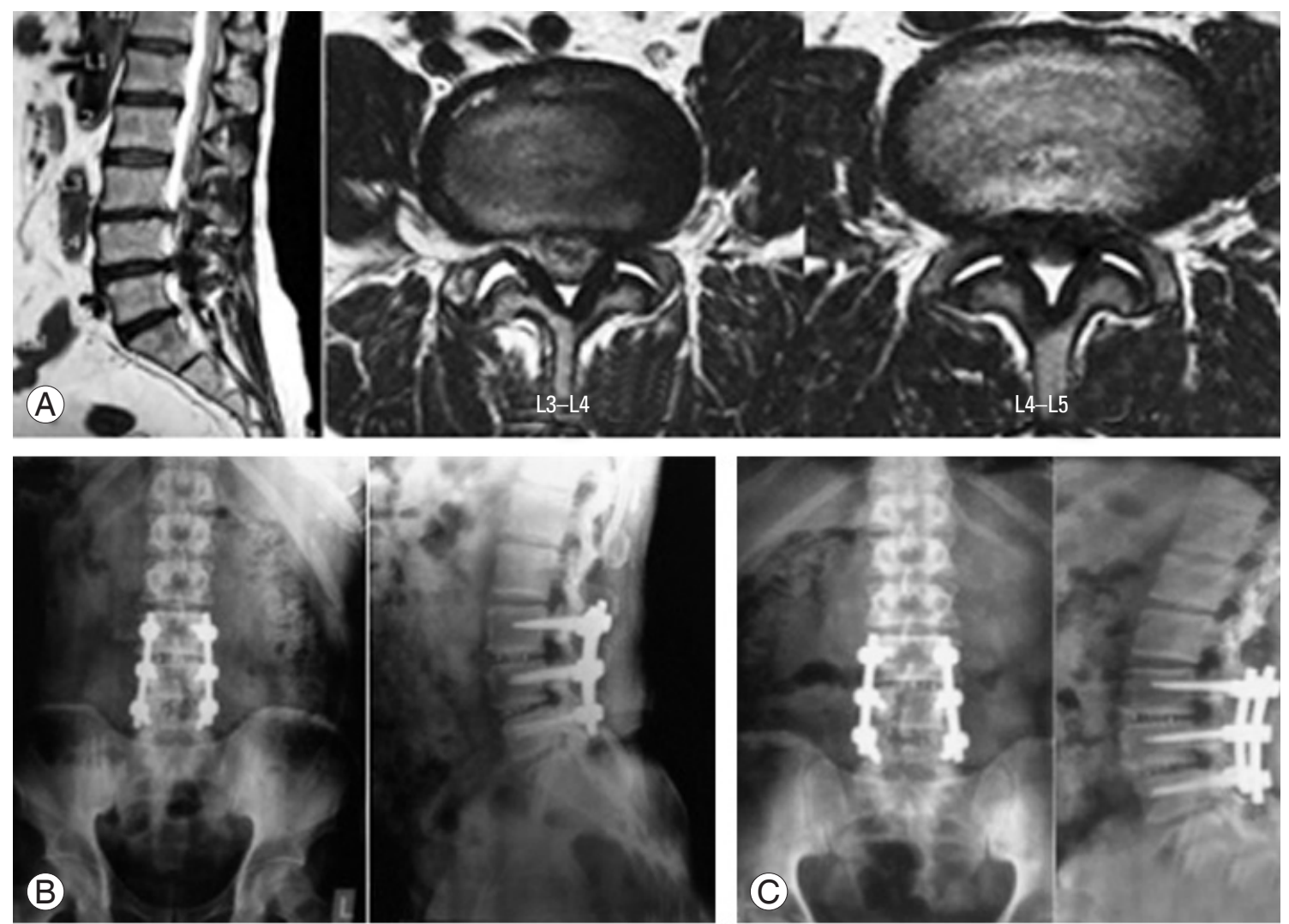

Fig. 2. (A) Preoperative magnetic resonance imaging (sagittal and axial images). (B) Immediate postoperative X-ray (AP and lateral views). (C) 5-Year postoperative X-ray (AP and lateral views). AP, anteroposterior. 

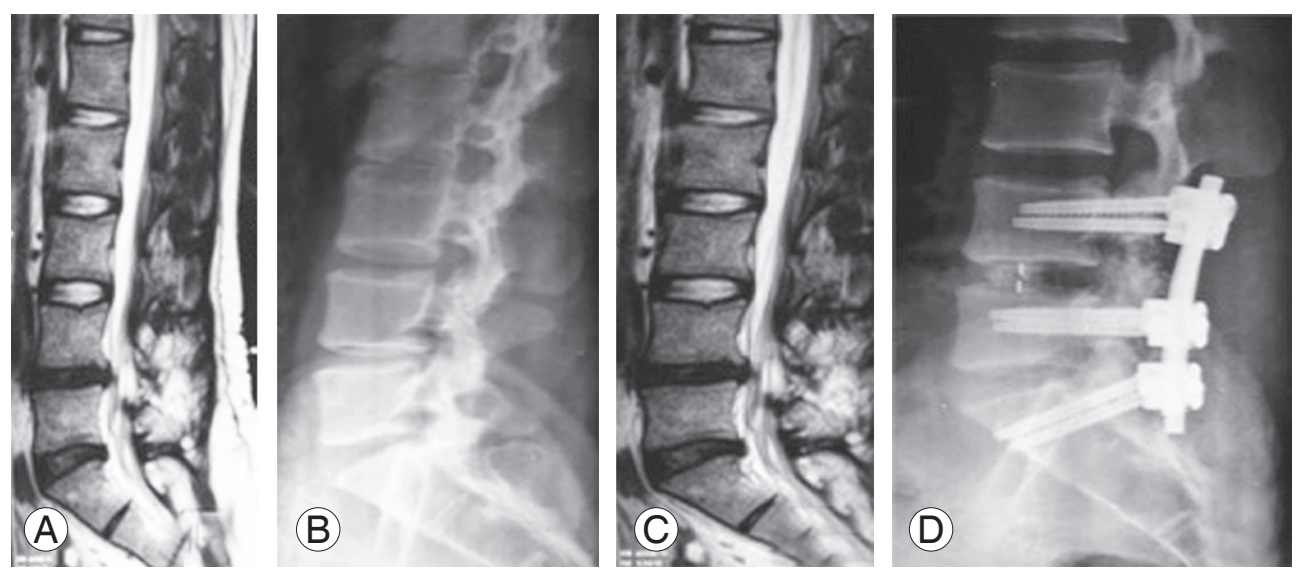

Fig. 3. (A) Postoperative MRI after failed L4/L5 microdiscectomy. (B, C) Postoperative MRI and X-ray after previous L4/L5 lumbar decompression. (D) Postoperative X-ray after L4-S1 instrumented fusion. MRI, magnetic resonance imaging.

ers, and one was a lawn tennis player. The average time for return to training and return to sports was 9 weeks and 3.5 months, respectively. Fig. 2 illustrates a case of cricketer undergoing L3-L5 transforaminal interbody fusion for L3-L4 and L4-L5 DDD with LCS (Fig. 2A, B) with good radiological outcome at 5 -year follow-up (Fig. 2C). Fig. 3 illustrates the case of a tennis player who underwent revision L4-S1 instrumented fusion for recurrent L4/L5 prolapsed intervertebral disc. Fusion was extended to S1 in order to address L5-S1 DDD as well. Cricketers as well as footballers are more prone to hyperextension injuries of the lumbar spine due to bone stress response of the pars interarticularis; however, there is a subtle difference between the patterns of injury. Stress fracture of the pars interarticularis is more often incomplete and unilateral (generally left) in cricketers indulging in fast bowling due to the asymmetric bone stress response. In footballers, the distribution of bone stress response is more likely to be symmetrical; hence, they are at an increased risk to have complete stress fracture of bilateral pars interarticularis [15]. In our unpublished series of eight young fast bowlers suffering with stress fractures of the pars interarticularis, all were managed successfully with nonoperative treatment. In the present series, one cricketer had L4/L5 prolapsed intervertebral disc with instability that underwent L4/L5 transforaminal lumbar interbody fusion. He returned to training at 6 weeks and returned to professional cricket playing within 6 months.

One of the marathon runners from this group had aseptic discitis who was managed nonoperatively as described previously. He took a longer duration to return to training (i.e., 16 weeks) but returned to competitive sports at
6 months. Excluding this patient, all other athletes in this group returned to training at 6 weeks, and they returned to sports at 3 months, respectively.

The outcomes of cervical spine fusion surgery for the treatment of single-level disc herniations have been well documented in the general population, with $90 \%-95 \%$ of the patients reporting excellent and good outcomes [16]. In athletes, the outcomes vary due to the daily high-velocity trauma and the unnatural stress on the cervical spine during play [17]. Hsu [18] analyzed the NFL players undergoing cervical fusion. They reported that $72 \%$ of players successfully returned to play for 29 games over a 2.8 year period, which was significantly greater than that of the nonoperative group ( $46 \%$ players), and successfully returned to play after treatment. Excellent outcomes, higher return-to-play rates, and longer careers were achieved by surgical treatment than those by nonoperative treatment. Maroon et al. [17] had reported similar results in their study of five elite athletes undergoing single-level ACDF surgery. Nevertheless, controversy remains regarding the management and return-to-play guidelines for athletes with multiple fusion levels. Along with the full participation of the athletes, the combined effort of team trainers and physiotherapists is essential. In the present study, two of 10 patients had cervical spine pathology. One patient was a wrestler who had C5-C6 prolapsed intervertebral disc and underwent $\mathrm{C} 5-\mathrm{C} 6 \mathrm{ACDF}$. The other patient was a marathon runner who had $\mathrm{C} 5-\mathrm{C} 6$ degenerative disc disease and underwent $\mathrm{C} 5-\mathrm{C} 6$ anterior cervical discectomy and disc replacement. Both these patients returned to training at 6 weeks and competitive sports at 3 months. The consensus to perform fusion in the wrestler was also 

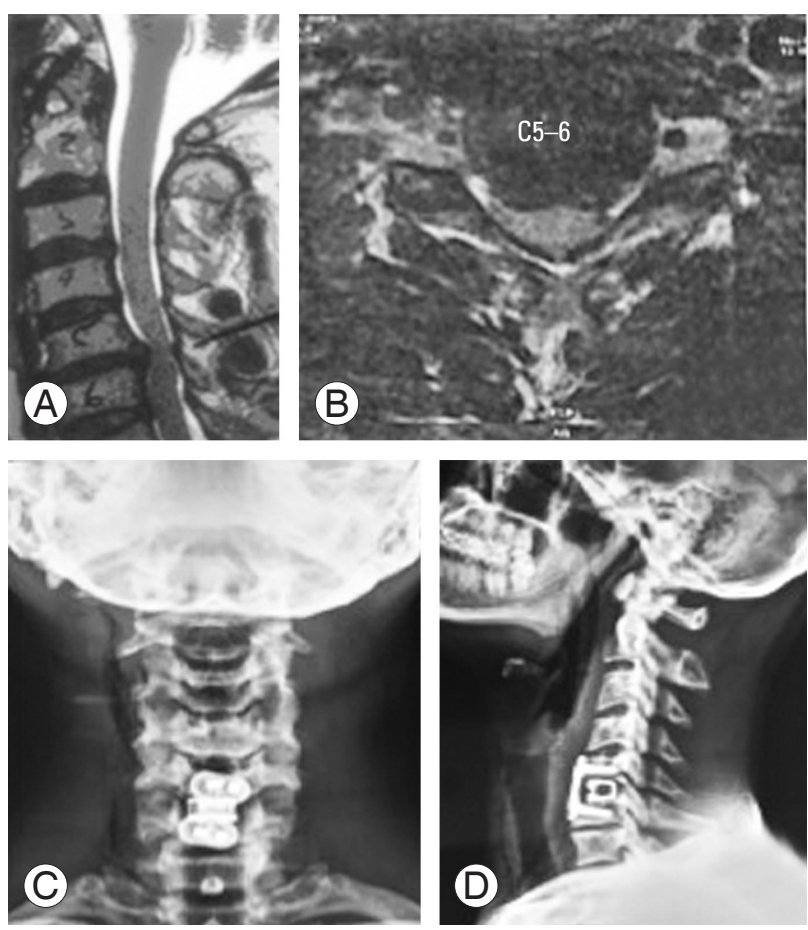

Fig. 4. (A, B) Preoperative magnetic resonance imaging (sagittal and axial views). (C, D) 3-Year follow-up X-ray (anteroposterior and lateral views).

based on the fact that he was involved in direct contact with his competitor during wrestling (Fig. 4).

Rehabilitation protocol for athletes undergoing surgery should be properly structured and aggressive so that they can go back to sports with their preinjury level of performance. Rehabilitation primarily aims at building a strong core and back extensor muscles, trunk stabilization with lumbar stretching, and sport-specific activities. Haus and Micheli [19] recommended that athletes generally return to activity at 3-6 months after nonoperative treatment and at 6-12 months after surgery. In another retrospective review of 59 professional and Olympic athletes undergoing lumbar microdiscectomy, Watkins et al. [20] found that $88 \%$ of the patients returned to active sports at an average period of 5.2 months following surgery. They emphasized the role of trunk stabilization and strengthening exercises for athletes to be able to return to their respective sports. Matsunaga et al. [21] reported a return-to-sport time of 7.5 weeks for athletes undergoing percutaneous discectomy and 17 weeks for those undergoing simple discectomy. Another study conducted by Abla et al. [22] recommended that the time for return to golf was 4-8 weeks after lumbar laminectomy and lumbar microdiscectomy, 2-3 months after anterior cervical fusion, and 6 months after lumbar fusion. In the present study, all athletes underwent the structured rehabilitation program as described and returned to sports at an average time of 45.6 weeks.

Although this study was a retrospective analysis, the findings shed light on the outcome of spinal surgeries in athletes and also highlight the importance of the need of the objective "return-to-sport" criteria for this different subset of population. The study also describes the structured rehabilitation program following spinal surgery in athletes. However, a prospective study with a larger number of participants is required to understand the effectiveness of the described rehabilitation program.

\section{Conclusions}

Spine surgery in patients involved in contact sports is safe and effective. Patients can return to training and playing contact sports as the presurgery level. Currently, there are no standardized guidelines for return to sports after spine injuries. An athlete needs to be symptom-free, with full range of motion and full strength before returning to sports.

\section{Conflict of Interest}

No potential conflict of interest relevant to this article was reported.

\section{References}

1. Rubin DI. Epidemiology and risk factors for spine pain. Neurol Clin 2007;25:353-71.

2. McCarroll JR, Miller JM, Ritter MA. Lumbar spondylolysis and spondylolisthesis in college football players: a prospective study. Am J Sports Med 1986;14:404-6.

3. Rubery PT, Bradford DS. Athletic activity after spine surgery in children and adolescents: results of a survey. Spine (Phila Pa 1976) 2002;27:423-7.

4. Hsu WK, McCarthy KJ, Savage JW, et al. The Professional Athlete Spine Initiative: outcomes after lumbar disc herniation in 342 elite professional athletes. Spine J 2011;11:180-6.

5. Hsu WK. Performance-based outcomes following lumbar discectomy in professional athletes in the National Football League. Spine (Phila Pa 1976) 2010;35:1247-51. 
6. Tones M, Moss N, Polly DW Jr. A review of quality of life and psychosocial issues in scoliosis. Spine (Phila Pa 1976) 2006;31:3027-38.

7. Weinstein JN, Tosteson TD, Lurie JD, et al. Surgical vs nonoperative treatment for lumbar disk herniation: the Spine Patient Outcomes Research Trial (SPORT): a randomized trial. JAMA 2006;296:244150.

8. Atlas SJ, Deyo RA, Keller RB, et al. The Maine Lumbar Spine Study, Part II. 1-year outcomes of surgical and nonsurgical management of sciatica. Spine (Phila Pa 1976) 1996;21:1777-86.

9. Anakwenze OA, Namdari S, Auerbach JD, et al. Athletic performance outcomes following lumbar discectomy in professional basketball players. Spine (Phila Pa 1976) 2010;35:825-8.

10. Schroeder GD, McCarthy KJ, Micev AJ, Terry MA, Hsu WK. Performance-based outcomes after nonoperative treatment, discectomy, and/or fusion for a lumbar disc herniation in National Hockey League athletes. Am J Sports Med 2013;41:2604-8.

11. Cahill KS, Dunn I, Gunnarsson T, Proctor MR. Lumbar microdiscectomy in pediatric patients: a large single-institution series. J Neurosurg Spine 2010;12:165-70.

12. Wang JC, Shapiro MS, Hatch JD, Knight J, Dorey FJ, Delamarter RB. The outcome of lumbar discectomy in elite athletes. Spine (Phila Pa 1976) 1999;24:570-3.

13. Shifflett GD, Hellman MD, Louie PK, Mikhail C, Park KU, Phillips FM. Return to golf after lumbar fusion. Sports Health 2017;9:280-4.

14. Bouras T, Korovessis P. Management of spondylolysis and low-grade spondylolisthesis in fine athletes: a comprehensive review. Eur J Orthop Surg Traumatol 2015;25 Suppl 1:S167-75.

15. Gregory PL, Batt ME, Kerslake RW. Comparing spondylolysis in cricketers and soccer players. Br J Sports Med 2004;38:737-42.

16. Bohlman HH, Emery SE, Goodfellow DB, Jones PK. Robinson anterior cervical discectomy and arthrodesis for cervical radiculopathy: long-term follow-up of one hundred and twenty-two patients. J Bone Joint Surg Am 1993;75:1298-307.

17. Maroon JC, El-Kadi H, Abla AA, et al. Cervical neurapraxia in elite athletes: evaluation and surgical treatment: report of five cases. J Neurosurg Spine 2007;6:356-63.

18. Hsu WK. Outcomes following nonoperative and operative treatment for cervical disc herniations in $\mathrm{Na}$ tional Football League athletes. Spine (Phila Pa 1976) 2011;36:800-5.

19. Haus BM, Micheli LJ. Back pain in the pediatric and adolescent athlete. Clin Sports Med 2012;31:423-40.

20. Watkins RG 4th, Williams LA, Watkins RG 3rd. Microscopic lumbar discectomy results for 60 cases in professional and Olympic athletes. Spine J 2003;3:100-5.

21. Matsunaga S, Sakou T, Taketomi E, Ijiri K. Comparison of operative results of lumbar disc herniation in manual laborers and athletes. Spine (Phila Pa 1976) 1993;18:2222-6.

22. Abla AA, Maroon JC, Lochhead R, Sonntag VK, Maroon A, Field M. Return to golf after spine surgery. J Neurosurg Spine 2011;14:23-30. 